\title{
A Case Report: Recurrent Hyperparathyroidism After Parathyroidectomy in a Primary Hyperparathyroidism Patient with Chronic Kidney Disease
}

\author{
Xia Qing Li ${ }^{1}$, Kai Jun Chen ${ }^{2}$, Yu Meng, ${ }^{1, *}$ \\ ${ }^{1}$ Department of Nephrology, the First Hospital Affiliated to Jinan University, Guangzhou, China \\ ${ }^{2}$ Department of Nephrology, People's Hospital of Longchuan County, Heyuan, China
}

Email address:

mengy@jnu.edu.cn (Yu Meng)

*Corresponding author

To cite this article:

Xia Qing Li, Kai Jun Chen, Yu Meng. A Case Report: Recurrent Hyperparathyroidism After Parathyroidectomy in a Primary

Hyperparathyroidism Patient with Chronic Kidney Disease. Science Journal of Clinical Medicine. Vol. 10, No. 2, 2021, pp. 42-46.

doi: $10.11648 /$ j.sjcm. 20211002.15

Received: May 18, 2021; Accepted: May 30, 2021; Published: June 10, 2021

\begin{abstract}
Background: Parathyroidectomy (PTX) is the definitive treatment of primary hyperparathyroidism (PHPT) for symptomatic patients. PTX for hyperparathyroidism is associated with the risk of recurrence; however, this has been mostly demonstrated in chronic kidney disease (CKD) patients with secondary hyperparathyroidism. The recurrent risk of hyperparathyroidism and renal outcomes after PTX in patients with PHPT has been rarely reported. Patient: In this article, we report a case of a 70-year-old man with previously diagnosed CKD, underwent a subtotal PTX for asymptomatic primary hyperparathyroidism induced by parathyroid hyperplasias. The PTX appears to resolve the calcium disturbance in this patient. However, at three months after the surgery, the diagnosis of recurrent hyperparathyroidism was made, based on a remarkably increased level of parathyroid hormone from $42.12 \mathrm{pg} / \mathrm{ml}$ to $270.9 \mathrm{pg} / \mathrm{ml}$. Over 2 years of follow-up, his renal function, as evaluated by serum creatinine levels, fluctuated modestly, but persist at a preoperative level of renal impairment. These data indicate that this patient with asymptomatic PHPT has experienced recurrent hyperparathyroidism with persistent renal dysfunction after PTX. Conclusion: The recurrent hyperparathyroidism after PTX in PHPT patients with a coexisting renal impairment appears more common than might be anticipated. We recommend that if hyperparathyroidism recurs or exacerbates by the persistent renal dysfunction after PTX, the potential benefits of PTX should be reconsidered and carefully weighed against the possible surgical risks.
\end{abstract}

Keywords: Hyperparathyroidism, Parathyroidectomy, Chronic Kidney Disease

\section{Introduction}

Primary hyperparathyroidism (PHPT) is a common endocrine disorder characterized by a disproportionate release of parathyroid hormone (PTH), resulting in hypercalcemia [1]. Up to $87 \%$ of cases are caused by parathyroid adenomas, while hyperplasia $(10 \% \sim 15 \%)$ and carcinoma $(1 \%)$ are less common [2]. PHPT may present as a symptomatic or asymptomatic disease. Patients with asymptomatic PHPT may have nonspecific symptoms (fatigue, weakness and depression), and measurable end-organ manifestations (decreased cortical bone mineral density, hypercalciuria, nephrocalcinosis, and reduced creatinine clearance [1]).
Despite its adverse effects on health, it is considered as an under-treated disease.

Parathyroidectomy (PTX) is the definitive treatment of PHPT for symptomatic patients [3]. Accumulating evidence show the benefits of PTX, including decreased progression of kidney disease and excellent long-term outcomes [3, 4]. According to the current guidelines, PTX is suggested in all symptomatic patients [5], and it is suggested to be more cost-effective than observation or pharmacologic therapy, even when the patient is considered asymptomatic [6]. PTX has also been recommended in 
asymptomatic patients with an estimated glomerular filtration rate (eGFR) less than $60 \mathrm{~mL} / \mathrm{min} / 1.73 \mathrm{~m}^{2}$, based on the speculation that PTX may be able to improve renal function [7].

Chronic kidney disease (CKD) is a major public health concern worldwide, leading to adverse outcomes of kidney failure [8, 9]. Considering the insults including nephrocalcinosis, hypercalciuria, tubulointerstitial inflammation [10], CKD is also recognized as a common complication in patients with PHPT, accounting for 13-19\% of this population. However, there are few reports on PHPT patients with previously diagnosed CKD. Indeed, some reports have demonstrated that PTX is associated with a risk of recurrent hyperparathyroidism in CKD patients with secondary hyperparathyroidism [11-13].

Some reports have indicated that PTX has limited benefits with respect to end-organ functions, such as cardiovascular $[14,15]$ and fracture outcomes [16]. However, there have been few reports on the renal outcomes and recurrent risk of hyperparathyroidism in patients with asymptomatic PHPT undergoing PTX.

We report here a rare case of recurrent hyperparathyroidism with persistent renal dysfunction in an asymptomatic PHPT patient with CKD after PTX.

\section{Case Report}

A 70-year-old man, with previously diagnosed chronic kidney disease (CKD), was admitted to our Nephrology Unit in December 2019, because of 1-month history of fatigue and weakness, with slight pitting edema of both lower extremities. His other medical history includes hypertension, coronary heart disease (CHD) and glaucoma. On physical examination, his thyroid gland was normal in size. The initially laboratory values in showed overtly hypercalcemic hyperparathyroidism (see Table 1): PTH $582.5 \mathrm{pg} / \mathrm{mL}$ (reference range, 12-88 $\mathrm{pg} / \mathrm{mL})$, serum calcium $2.91 \mathrm{mmol} / \mathrm{L}(2-2.8 \mathrm{mmol} / \mathrm{L})$, serum phosphate $0.64 \mathrm{mmol} / \mathrm{L}(0.96-1.62 \mathrm{mmol} / \mathrm{L})$, and serum creatinine $210.2 \mathrm{umol} / \mathrm{L} \quad(32-106 \mathrm{umol} / \mathrm{L}$, estimated glomerular filtration rate [eGFR] $26.64 \mathrm{~mL} / \mathrm{min} / 1.73 \mathrm{~m}^{2}$. Renal ultrasound was performed, and suggested multiple cysts in both kidneys, but did not show kidney stone. The ultrasound of thyroid and parathyroid, and 99mTc-MIBI parathyroid SPECT/CT were also performed (the results are shown in figures 1 and 2). The whole-body bone scan showed multiple active bone lesions, including ribs, spinal cones, and bilateral shoulder joints and elbow joints.

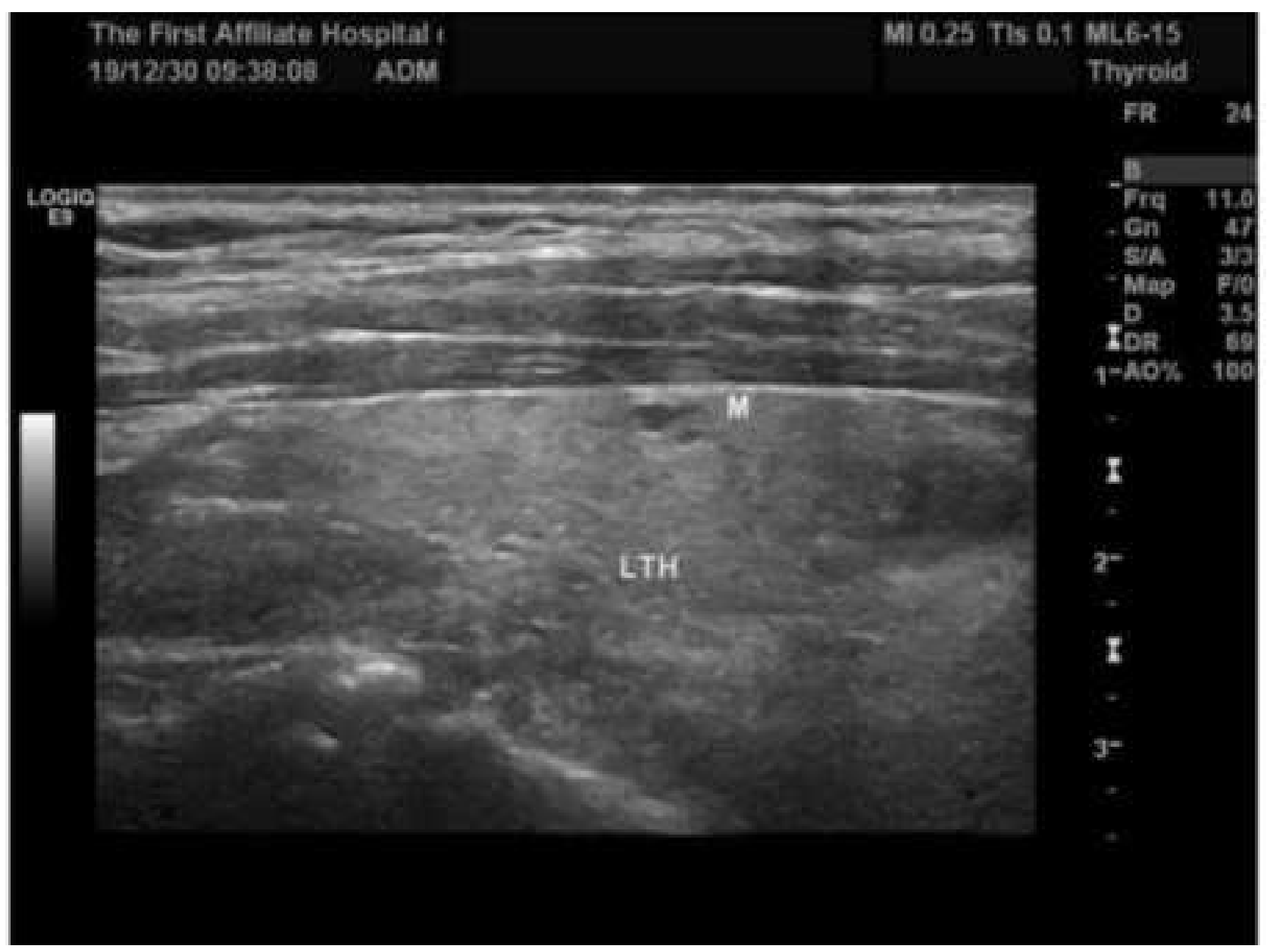

Ultrasound of thyroid and parathyroid indicated the presence of nodular goiter and bilateral parathyroid space-occupying lesion.

Figure 1. Ultrasound of thyroid and parathyroid. 


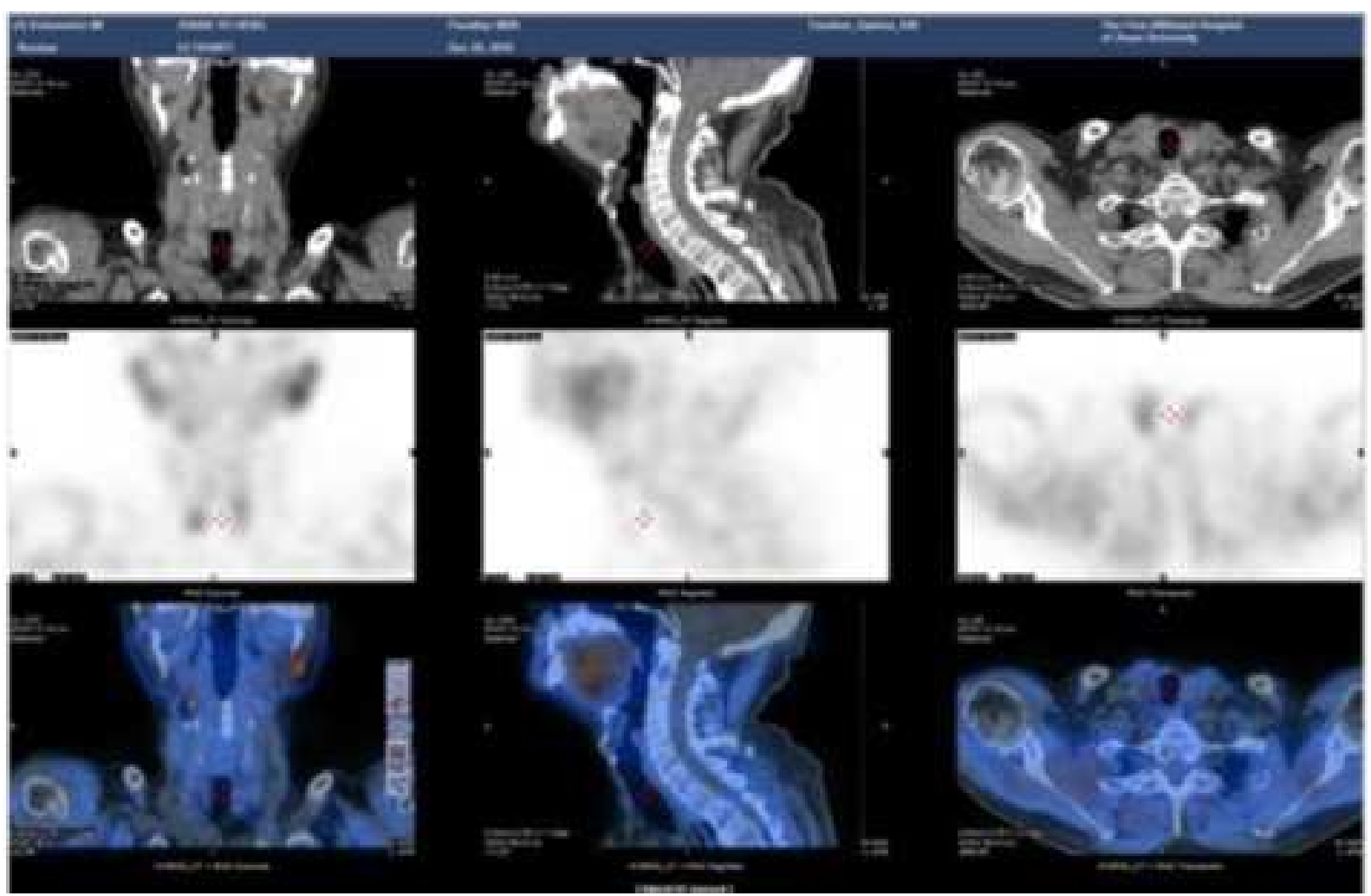

Figure 2. Scintigraphy and single photon emission computed tomography/computed tomography (SPECT/CT) of parathyroid.

99mTc-MIBI scintigraphy showed high tracer uptake in the thyroid and the lesion became unclear with delayed imaging, but a solid mass with high tracer uptake in the bilateral parathyroid glands.

Table 1. Evaluation of biochemical values before and after parathyroidectomy.

\begin{tabular}{|c|c|c|c|c|c|c|}
\hline & \multicolumn{6}{|l|}{2019} \\
\hline & Dec-19 & Dec-24 & Dec-25 & Dec-26 & Dec-27 & Dec-31 \\
\hline $\mathrm{PTH}, \mathrm{pg} / \mathrm{ml}$ & 582.5 & & 686.9 & & & 721.7 \\
\hline Serum calcium, $\mathrm{mmol} / \mathrm{L}$ & 2.91 & 3.02 & 2.91 & & 2.9 & 2.77 \\
\hline Urinary calcium, $\mathrm{mmol} / \mathrm{L}$ & & & & 4.53 & & \\
\hline Phosphate, $\mathrm{mmol} / \mathrm{L}$ & & & 0.62 & & & \\
\hline 25-Hydroxyvitamin D3, ng/ml & 0.64 & & 16 & & 0.76 & 0.76 \\
\hline $\mathrm{sCr}, \mathrm{umol} / \mathrm{L}$ & 210.2 & 257 & 236 & & 191.4 & 205 \\
\hline $\mathrm{BUN}, \mathrm{mmol} / \mathrm{L}$ & & 14.77 & 12.32 & & 9.58 & 6.16 \\
\hline
\end{tabular}

Table 1. Continued.

\begin{tabular}{|c|c|c|c|c|c|c|c|c|c|}
\hline & \multicolumn{7}{|l|}{2020} & \multirow{2}{*}{$\begin{array}{l}2021 \\
\text { Feb-8 }\end{array}$} & \multirow{2}{*}{$\begin{array}{l}\text { Reference } \\
\text { Range }\end{array}$} \\
\hline & Jan-3 & Jan-4 & Jan-5 & Jan-6 to 17 & Feb-21 & Mar-20 & Oct-22 & & \\
\hline $\mathrm{PTH}, \mathrm{pg} / \mathrm{ml}$ & 42.12 & 82.62 & 85.64 & & 168.9 & 270.9 & 168.9 & 178.6 & $12-88$ \\
\hline Serum calcium, mmol/L & 2.22 & 2.09 & 2 & $2.04-2.44$ & 2.47 & 2.48 & 2.21 & 2.5 & $2-2.8$ \\
\hline Urinary calcium, $\mathrm{mmol} / \mathrm{L}$ & & & & & & & & & $2-2.8$ \\
\hline Phosphate, $\mathrm{mmol} / \mathrm{L}$ & & & & & & & & & $0.96-1.62$ \\
\hline 25-Hydroxyvitamin D3, ng/ml & & 0.69 & & $0.66-0.74$ & 0.92 & 0.82 & 0.95 & 0.96 & $10-30$ \\
\hline $\mathrm{sCr}, \mathrm{umol} / \mathrm{L}$ & & 221.4 & 250 & $204.9-254.6$ & 217.10 & 233 & 217.1 & 246.7 & $32-106$ \\
\hline $\mathrm{BUN}, \mathrm{mmol} / \mathrm{L}$ & & 7.25 & 8.01 & $6.74-7.98$ & 11.85 & 10.48 & 11.85 & 12.18 & $1.5-7.5$ \\
\hline
\end{tabular}

PTH, parathyroid hormone; sCr, Serum creatinine; BUN, blood urea nitrogen

He subsequently underwent a "subtotal PTX under endoscopy and bilateral recurrent laryngeal nerve exploration" on January 2, 2020. During surgical exploration, enlarged right inferior $(2 \times 1.3 \times 1.3 \mathrm{~cm})$, left superior $(1 \times 0.6 \times 0.8 \mathrm{~cm})$ and left inferior $(1.1 \times 0.6 \times 1.1 \mathrm{~cm})$ parathyroid glands were resected. The diagnosis of asymptomatic PHPT could be made, based on his nonspecific symptoms and a postoperative pathological result of parathyroid hyperplasias (Figure 3). On the day of surgery (see Table 1), his serum calcium was $2.63 \mathrm{mmol} / \mathrm{L}$, and bone mineral density measurement showed osteoporosis (T-score: -3.2 whole-body bone). The PTX contributed to a PTH nadir of $42.12 \mathrm{pg} / \mathrm{ml}$, and his serum calcium normalized 
to $2.2 \mathrm{mmol} / \mathrm{L}$ within 24 hours after surgery. Since the PTX, the patient's hypercalcemia was managed conservatively, fluctuating from a low of $2.22 \mathrm{mmol} / \mathrm{L}$ to a high of 2.50 $\mathrm{mmol} / \mathrm{L}$. However, there was a tendency of gradually increasing postoperative PTH, and the PTH increased to 85.64 $\mathrm{pg} / \mathrm{ml}$ on the fourth day after surgery (Figure 4). During the follow-up evaluation in March 2020, hyperparathyroidism had recured based on the laboratory findings of PTH of 270.9 $\mathrm{pg} / \mathrm{Ml}$. In the following year, although was a mild recovery of PTH with pharmacologic intervention, it still maintained at a high level, along with a persistent renal dysfunction.
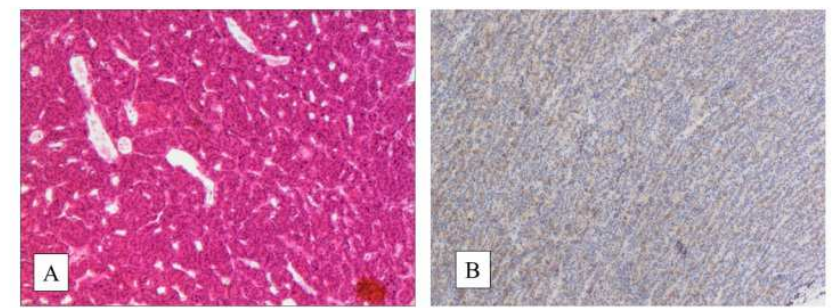

Figure 3. Postoperative histopathological findings. (A) The pathological results showed that parathyroid hyperplasias; (B) Immunohistochemical staining showed that the cells were positive for chromogranin A, and negative for thyroglobulin, thyroid transcription factor-1 and calcitonin, synaptophysin, with a Ki-67 index of $10 \%$.

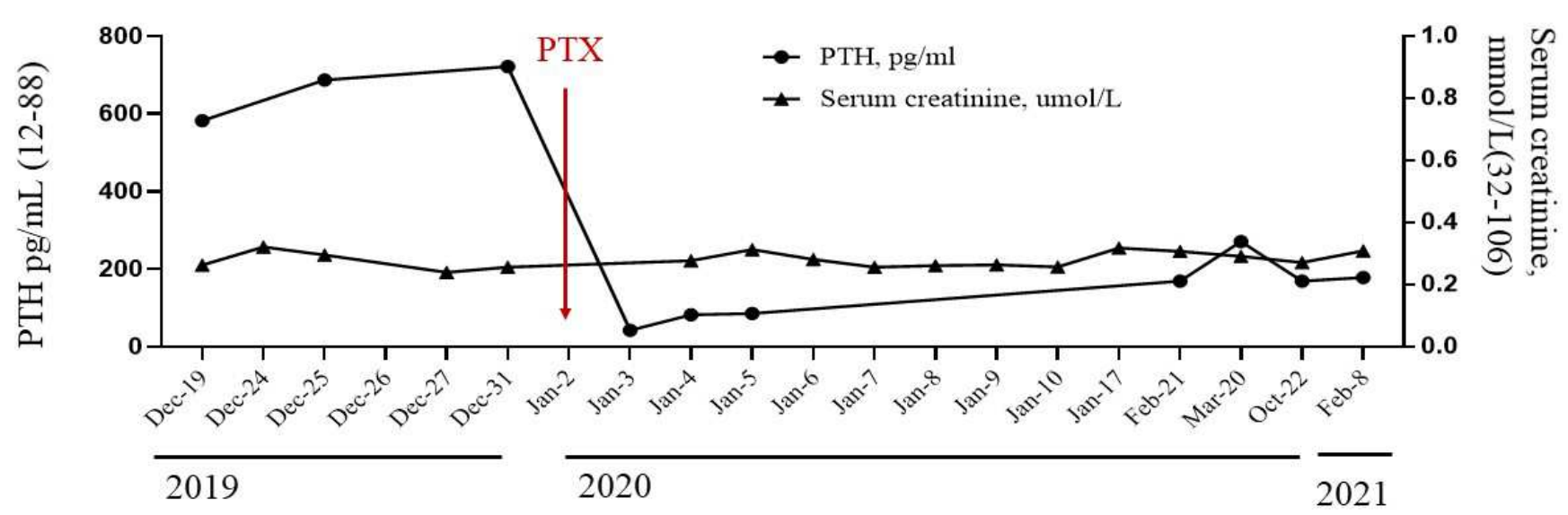

Figure 4. Time course of patient's parathyroid hormone and serum creatinine levels before and after parathyroidectomy.

\section{Discussion}

Our case shows several findings rarely seen in the clinical setting. First, hypercalcemia and hypophosphatemia occurred simultaneously in a patient with CKD; Second, no beneficial effects of PTX on renal function were shown in this patient; Third, the hyperparathyroidism had recurred after successful PTX.

In PHPT patients with a coexisting renal impairment, the results of renal function after PTX are still controversial. Although some retrospective studies show benefits to kidney function of patients undergoing PTX [4, 10, 17], other studies reported that PTX is associated with acute and permanent renal impairment in PHPT patients [18-20]. A prospective study of 494 patients undergoing PTX for PHPT reported a robust eGFR decrease of $21 \mathrm{~mL} / \mathrm{min} / 1.73 \mathrm{~m}^{2}$ in the acute period, and $48.8 \%$ of patients fulfilled the KDIGO criteria for AKI [19]. However, the renal outcome of this patient conflicts with those of current students. We did not observe an obvious change in postoperative renal functions in this patient. Compared with preoperative results, PTX neither acutely lead to a further exacerbation nor an improvement in renal function. As is shown in figure 1 , within the 2 years of follow-up, $\mathrm{sCr}$ fluctuated modestly, but finally preserved to the preoperative level of renal impairment. The PTX appears to resolve the calcium disturbance in this patient. However, recurrent hyperparathyroidism occurred in this patient during short-term follow up, and this hyperparathyroidism was maintained for a year, presumably due to the persistent renal dysfunction.
It is previously reported that PTX is associated with a risk of recurrent hyperparathyroidism in CKD patients with secondary hyperparathyroidism. In a previous case report, Lin et al described a hemodialysis patient who suffered from recurrent secondary hyperparathyroidism within 1 year after PTX [12]. In another report, Sippel et al have described a case of recurrent secondary hyperparathyroidism resulting from hyperplasia of forearm graft fragments in a patient with the end-stage renal disease after subtotal PTX [11] However, there are few reports of recurrent hyperparathyroidism after PTX in asymptomatic PHPT patients with CKD. The results raise the possibility that the hyperparathyroidism induced by secondary causes was suppressed or got silenced before the parathyroid glands were surgically removed in this patient.

A combination of factors, including old age, preoperative comorbidities (hypertension and CHD), kidney cysts, and recurrent hyperparathyroidism, may be responsible for post-operative renal dysfunction in this patient. A recent study suggested that the beneficial effect of PTX is more pronounced in patients with age $<65$ years, non-CKD, and those without hypertension [10]. These facts raise a series of questions: Whether each patient with the diagnosis of PHPT requires PTX? How should the benefits of surgery be weighed against the risks? What is the therapeutic difference between parathyroid hyperplasia and other types of PHPT (thyrophyma and thyroid cancer)? Therefore, this case helps to offer novel insight into the revision of PHPT treatment guidelines. A 'Renal risk score' for identifying those at risk of post-operative kidney injury [20] may be necessary for PHPT 
treatment in the future.

\section{Conclusion}

We report an asymptomatic PHPT patient with CKD who has experienced recurrent hyperparathyroidism and persistent renal function after successful PTX. Therefore, the recurrent hyperparathyroidism after PTX in PHPT patients with a coexisting renal impairment appears more common than might be anticipated. We would advocate for more studies on the efficacy of PTX for asymptomatic PHPT patients, and recommend that if hyperparathyroidism recured or exacerbated by the persistent renal dysfunction after PTX, the potential benefits of PTX will be reconsidered and carefully weighed against the possible surgical risks.

\section{Conflict of Interest}

The authors declare that they have no competing interests.

\section{Author Contribution}

L. X. Q wrote the manuscript and prepared figures. C. K. J collected the data and performed data analysis; M. Y. edited and revised the manuscript. All authors read and approved the submitted version.

\section{Funding}

This work was supported by grants from the Natural Science Foundation of Guangdong Province (2019A1515010176 and 2018A030313527).

\section{References}

[1] C. Y. Zhu, C. Sturgeon, M. W. Yeh, Diagnosis and Management of Primary Hyperparathyroidism, JAMA. 2020 Mar 24; 323 (12): 1186-1187.

[2] R. A. DeLellis, P. Mazzaglia, S. Mangray, Primary hyperparathyroidism: a current perspective, Arch Pathol Lab Med. 2008 Aug; 132 (8): 1251-1262.

[3] E. A. Alore, J. W. Suliburk, D. J. Ramsey, et al., Diagnosis and Management of Primary Hyperparathyroidism Across the Veterans Affairs Health Care System, JAMA Intern Med. 2019 Jul 15.

[4] F. Tassone, A. Guarnieri, E. Castellano, et al., Parathyroidectomy Halts the Deterioration of Renal Function in Primary Hyperparathyroidism, J Clin Endocrinol Metab. 2015 Aug; 100 (8): 3069-3073.

[5] S. M. Wilhelm, T. S. Wang, D. T. Ruan, et al., The American Association of Endocrine Surgeons Guidelines for Definitive Management of Primary Hyperparathyroidism, JAMA Surg. 2016 Oct 1; 151 (10): 959-968.
[6] K. Zanocco, P. Angelos, C. Sturgeon, Cost-effectiveness analysis of parathyroidectomy for asymptomatic primary hyperparathyroidism, Surgery. 2006 Dec; 140 (6): 874-881; discussion 881-872.

[7] C. Verdelli, S. Corbetta, MECHANISMS IN ENDOCRINOLOGY: Kidney involvement in patients with primary hyperparathyroidism: an update on clinical and molecular aspects, Eur J Endocrinol. 2017 Jan; 176 (1): R39-R52.

[8] K. Bruck, V. S. Stel, G. Gambaro, et al., CKD Prevalence Varies across the European General Population, J. Am. Soc. Nephrol. 2016 Jul; 27 (7): 2135-2147.

[9] 2015 USRDS Annual Data Report: Epidemiology of Kidney Disease in the United States, 2015.

[10] C. C. Liang, H. C. Yeh, Y. C. Lo, et al., Parathyroidectomy slows renal function decline in patients with primary hyperparathyroidism, J. Endocrinol. Invest. 2021 Apr; 44 (4): 755-763.

[11] R. S. Sippel, J. Bianco, H. Chen, Radioguided parathyroidectomy for recurrent hyperparathyroidism caused by forearm graft hyperplasia, J Bone Miner Res. 2003 May; 18 (5): 939-942.

[12] N. Lin, Y. Fang, C. Yu, et al., A useful treatment for recurrent secondary hyperparathyroidism after Parathyroidectomy: A case report, Asian J Surg. 2020 Apr; 43 (4): 573-574.

[13] J. Stratton, M. Simcock, H. Thompson, et al., Predictors of recurrent hyperparathyroidism after total parathyroidectomy in chronic renal failure, Nephron Clin Pract. 200395 (1): c15-22.

[14] D. J. McMahon, A. Carrelli, N. Palmeri, et al., Effect of Parathyroidectomy Upon Left Ventricular Mass in Primary Hyperparathyroidism: A Meta-Analysis, J Clin Endocr Metab. 2015 Dec; 100 (12): 4399-4407.

[15] C. Dural, A. K. Okoh, A. Seicean, et al., A pilot study investigating the effect of parathyroidectomy on arterial stiffness and coronary artery calcification in patients with primary hyperparathyroidism, Surgery. 2016 Jan; 159 (1): 218-224.

[16] F. Zheng, H. Zhou, N. Li, et al., Skeletal effects of failed parathyroidectomy, Surgery. 2018 Jan; 163 (1): 17-21.

[17] C. G. Nair, M. Babu, P. Jacob, et al., Renal dysfunction in primary hyperparathyroidism; effect of Parathyroidectomy: A retrospective Cohort Study, Int J Surg. 2016 Dec; 36 (Pt A): 383-387.

[18] M. Peacock, Primary hyperparathyroidism and the kidney: biochemical and clinical spectrum, J Bone Miner Res. 2002 Nov; 17 Suppl 2 N87-94.

[19] M. Belli, R. M. Martin, M. D. G. Brescia, et al., Acute and long-term kidney function after parathyroidectomy for primary hyperparathyroidism, PLoS One. 202015 (12): e0244162.

[20] R. J. Egan, F. Dewi, R. Arkell, et al., Does elective parathyroidectomy for primary hyperparathyroidism affect renal function? A prospective cohort study, Int J Surg. 2016 Mar; 27 138-141. 